\title{
Characterizing the transcriptome and microsatellite markers for almond (Amygdalus communis L.) using the Illumina sequencing platform
}

\author{
Linsen Zhang, Xiaoni Yang, Xiangning Qi, Chunhui Guo and Zhaobin Jing*
}

\begin{abstract}
Background: The almond tree (Prunus amygdalus Batsch) is an important nut tree grown in subtropical regions that produces nutrient-rich nuts. However, a paucity of genomic information and DNA markers has restricted the development of modern breeding technologies for almond trees.

Results: In this study, almonds were sequenced with Illumina paired-end sequencing technology to obtain transcriptome data and develop simple sequence repeats (SSR) markers. We generated approximately 64 million clean reads from the various tissues of mixed almonds, and a total of 42,135 unigenes with an average length of $988 \mathrm{bp}$ were obtained in the present study. A total of 27,586 unigenes (57.7\% of all unigenes generated) were annotated using several databases. A total of 112,812 unigenes were annotated with the Gene Ontology (GO) database and assigned to 82 functional sub-groups, and 29,075 unigenes were assigned to the KOG database and classified into 25 function classifications. There were 9470 unigenes assigned to 129 Kyoto Encyclopaedia of Genes and Genomes (KEGG) pathways from five categories in the KEGG pathway database. We further identified 8641 SSR markers from 48,012 unigenes. A total of 100 SSR markers were randomly selected to validate quality, and 82 markers could amplify the specific products of $A$. communis $L$., whereas 70 markers were successfully transferable to five species (A. ledebouriana, A. mongolica, A. pedunculata, A. tangutica, and A. triloba).
\end{abstract}

Conclusions: Our study was the first to produce public transcriptome data from almonds. The development of SSR markers will promote genetics research and breeding programmes for almonds.

Keywords: Prunus amygdalus Batsch, Transcriptome, Functional classification, SSR markers

\section{Background}

The almond (Prunus amygdalus Batsch) is one of the world's most important nut crops. The almond belongs to the genus Prunus, subgenus Amygdalus [1]. Almonds are widely used in food production and have high nutritional value [2]. Most Amygdalus wild species are highly tolerant of cold, drought and salt [3]. Almonds are a typical outbreeding species with gametophyte self-incompatibility [4]. They are also an antioxidant source [5] and an important germplasm resource in breeding programmes [6]. Because almonds have high nutritional value, past research

\footnotetext{
* Correspondence: jingzhaobin@nwsuaf.edu.cn
College of Horticulture, Northwest A\&F University, Yangling 712100, Shaanxi,

*Correspondence: jingzhaobin@nwsuaf.edu.cn
College of Horticulture, Northwest A\&F University, Yangling 712100, Shaanxi, People's Republic of China
}

has largely focused on physicochemical properties, seed nutrients and oil extraction $[2,7,8]$. However, despite high economic and nutritional values, genome and genetic resources regarding almonds are scarce, which has restricted the development of modern breeding technologies for amonds.

olecular markers are important tools used in evaluting genetic diversity among plant species and plant olecular breeding (marker-assisted breeding). In alnd trees, expressed sequence tags (ESTs) have been loped, but currently, there are only 3926 ESTs and loci available for almond in the NCBI GenBank database. Therefore, the number of ESTs is not sufficient to address almond tree molecular breeding development. 
Simple sequence repeats (SSRs) are widely used in studies on genetic diversity and relationships of plants because they are highly polymorphic, co-dominant and reproducible [9]. Moreover, SSRs markers could be used in the QTL mapping of important agronomical traits loci and marker-assisted selection in almond trees [10-13]. Presently, however, only a few SSRs have been reported in almonds [14-16]. SRAP and AFLP markers have been used to study genetic diversity and relationships in wild Amygdalus species [2]. A transcriptome is the complete collection of RNA that includes the full range of mRNA, tRNA, rRNA, and other noncoding RNA molecules expressed by one or a group of cells, organs or tissues in a particular environment or a specific developmental stage. The Illumina paired-end sequencing technique has been widely used for transcriptome analysis in plants [10, 17-19].

Transcriptome sequencing is helpful in developing SSR molecular markers, as it is reliable and efficient [20]. However, transcriptomic and associated molecular markers in almonds have not yet been reported. In this study, Illumina sequencing technology was used to analyse the transcriptome of almonds and develop EST-SSR markers. To our knowledge, this is the first study to characterize the almond transcriptome. Our study will provide a foundation for almond molecular biology and molecular breeding of almonds.

\section{Results}

Transcriptome generation and de novo assembly

A total of 66,668,192 raw reads were generated from our Illumina HiSeq ${ }^{\text {rm }} 2000$ paired-end sequencing of almonds.
The total length of the reads was approximately 10 Gigabase pairs (Gb); a total of 64,924,070 (97.38\%) high-quality clean reads $(1,440,780,4.32 \%)$ as well as low-quality reads $(1,702,602,2.55 \%)$ were collected after removing the adapter. All high-quality reads were assembled using the Trinity program, and a total of 42,135 unigenes with an average length of $988 \mathrm{bp}$ and an N50 length of $1714 \mathrm{bp}$ were obtained. The unigenes ranged from $201 \mathrm{bp}$ to 15,555 bp (Table 1). As shown in Fig. 1, 27,723 unigenes (65.80\%) ranged from 201 to $1000 \mathrm{bp}, 8699$ unigenes (20.65\%) were longer than $1000 \mathrm{bp}$ and 5713 unigenes (13.56\%) were longer than $2000 \mathrm{bp}$. The coverage percentage of read-blasted unigenes was 55.74\% (more than 10 reads), $19.17 \%$ (more than 100 reads), and $25.09 \%$ (more than 1000 reads) (Additional file 1). All of the raw data were submitted to the NCBI database (accession number: PRJNA347906).

\section{Functional annotation}

The function annotation of the unigenes was performed in the Nr, Swiss-Prot, Kyoto Encyclopaedia of Genes and Genomes (KEGG) and KOG databases by BLASTX (Evalue $\left.<10^{-5}\right)$. A total of 27,586 unigenes $(57.7 \%$ of all unigenes) were annotated. A total of 29,315 unigenes (69.6\% of all unigenes) of the largest match were annotated in the $\mathrm{Nr}$ database, followed by the Swiss-Prot $(20,642,49.0 \%)$, KOG $(16,794,39.9 \%)$ and KEGG $(11,055$, 26.2\%) databases (Fig. 2). A total of 12,671 (42.5\%) unigenes were not annotated in the four databases, indicating that these unigenes may be novel genes (Table 1). For the annotated unigenes in the $\mathrm{Nr}$ databases, the homologous

Table 1 Summary of transcriptome data for persimmon

\begin{tabular}{ll}
\hline Item & Number \\
\hline 1. Raw sequences and Assembly statistics & $66,668,192$ \\
Total reads & $10,000,228,800$ \\
Total nucleotides (bp) & $46.77 \%$ \\
GC content percentage & $42,135(988 ; 1714 ; 201-15,555)$ \\
Total unigenes (average length; N50; min-max length) & $14.8(0-7109)$ \\
Mean RPKM value of unigenes (min-mix RPKM value) & $29,315(69.6)$ \\
2. Bioinformatics annotations of mango fruit unigenes & $20,642(49.0)$ \\
Gene annotation against Nr (\%) & $11,055(26.2)$ \\
Gene annotation against Swiss-Prot (\%) & $16,794(39.9)$ \\
Gene annotation against KEGG (\%) & $29,464(69.9)$ \\
Gene annotation against KOG (\%) & $12,671(30.1)$ \\
All annotation genes (\%) & 51,$202 ;$ \\
Without annotation genes (\%) & 24,$483 ;$ \\
GO Ontology (\%) & 37,$133 ;$ \\
Biological process category & \\
Cellular component category & \\
Molecular function category & 2 \\
\hline
\end{tabular}




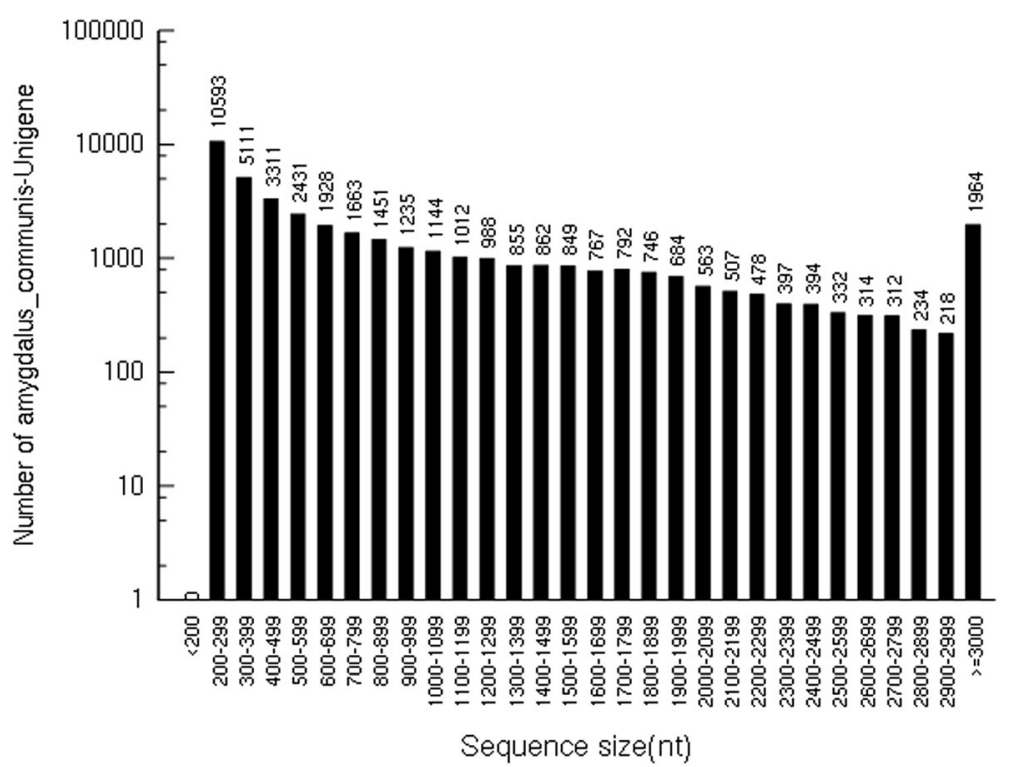

Fig. 1 Length distributions of the unigenes. The $x$-axis indicates a different sequence size, and the $y$-axis indicates the unigene numbers of a specific sequence size

sequences belonging to the species were analysed. The ten top-hit species were Prunus mume (18,097, 61.73\%), Malus domestica (15,80, 5.40\%), Theobroma cacao (1238, 4.22\%), Pyrus $x$ bretschneideri (1132, 3.86\%), Prunus persica (1061, 3.46\%), Gossypium arboreum (853, 2.90\%), Brassica napus (710, 2.42\%), Medicago truncatula (682, $2.33 \%)$, Fragaria vesca subsp. vesca $(665,2.27 \%)$, Morus notabilis $(311,1.06 \%)$, and others $(3029,10.34 \%)$.

Functional classification by gene ontology (GO) and KOG To further evaluate the functions of the almond unigenes, we used GO assignments to classify the almond unigene functions. A total of 112,812 unigenes were assigned to 82 functional sub-groups. Of the three ontology categories, the largest was biological process $(51,202$ unigenes), followed by cellular component (37,133 unigenes) and molecular function (24,483 unigenes) (Fig. 3). For the biological process group, the most frequent process was metabolic process $(11,647,22.75 \%)$, followed by cellular process $(10,967,21.42 \%)$. Cell $(8581,23.11 \%)$ and cell part $(8581,23.11 \%)$ were the most highly represented groups in the cellular component category. For the molecular function category, binding (11,139, $45.50 \%)$ and catalytic activity $(10,346,27.86 \%)$ represented the greatest proportion. The GO classifications of the unigenes are listed in Additional file 2.

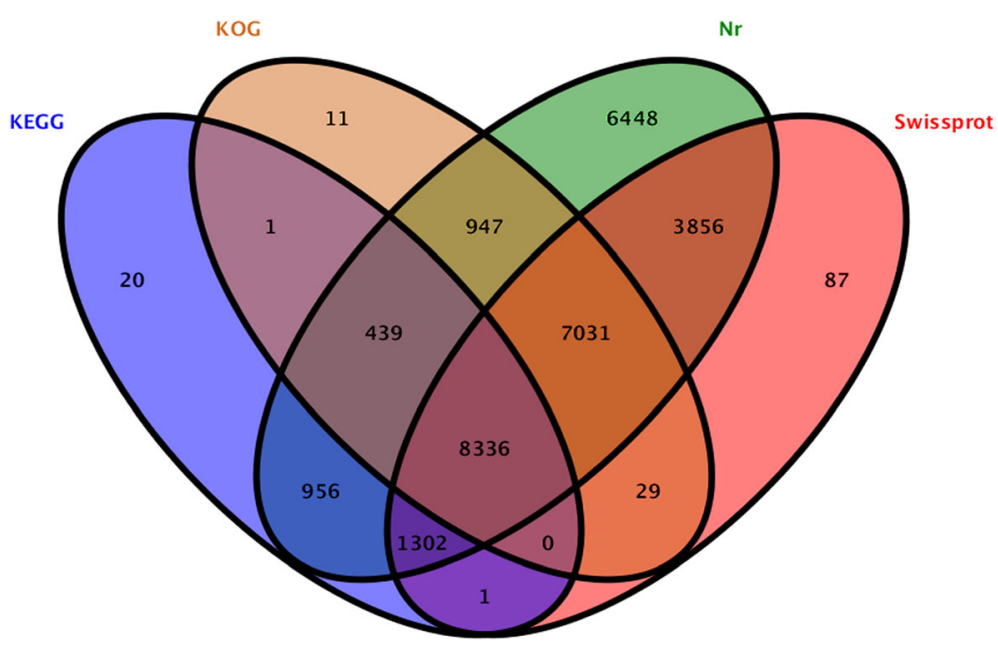

Fig. 2 Venn diagram of Nr, Swiss-Prot, KOG and KEGG databases. Venn diagram showing homology sequence numbers of the unigenes in the Nr, Swiss-Prot, GOG and KEGG databases 


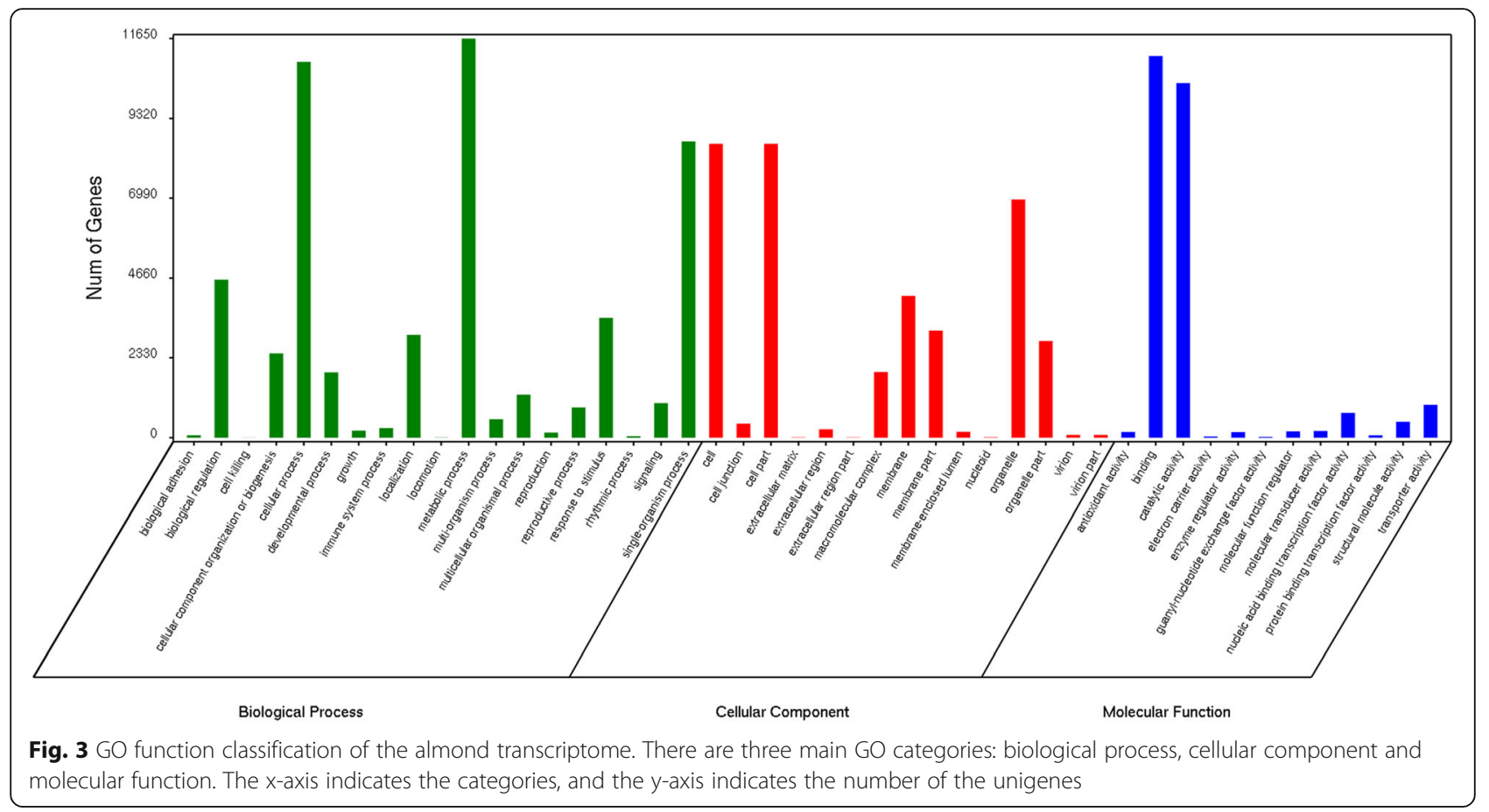

KOG classifications were searched based on a BLAST search against the KOG database. A total of 29,075 unigenes were classified into 25 function classifications (Fig. 4). For the 25 KOG categories, the general function prediction was the largest group (5895, 20.28\%); posttranslational modification, protein turnover, chaperones $(3311,11.39 \%)$, and signal transduction mechanisms
(3152, 10.84\%) had high percentages, and 15,012 unigenes were assigned to other functional categories (Additional file 3).

\section{Functional classifications by KEGG}

The pathway annotations were used to analyse the biological functions of genes. In this study, 9470 unigenes

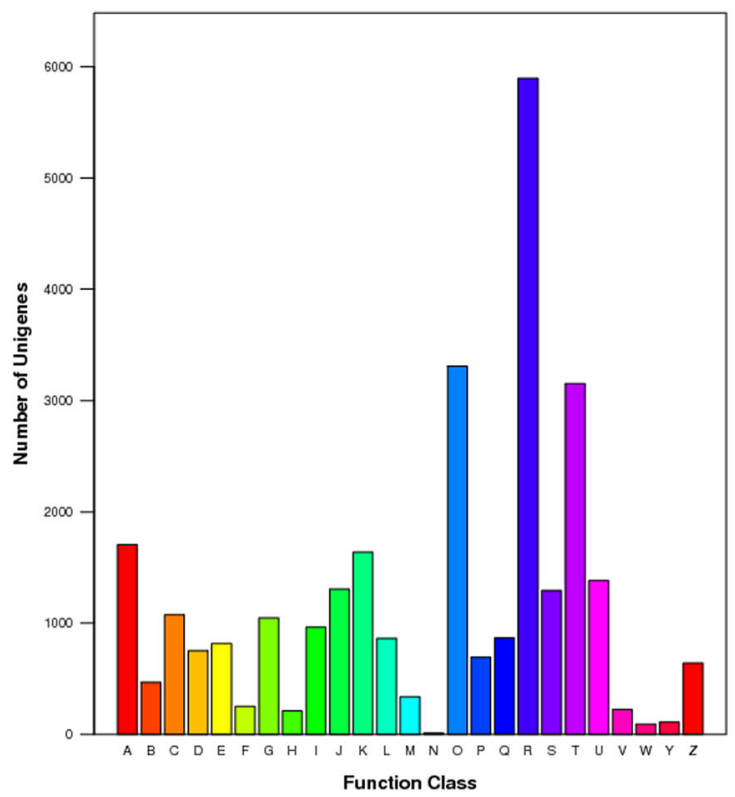

A: RNA processing and modification

$\mathrm{B}:$ Chromatin structure and dynamics

C: Energy production and corrversion

D: Coll cycle control, cell division, chromosome partitioning

E: Amino acid transport and motabolism

F: Nucleotide transport and metabolism

G: Carbohydrate transport and metabolism

$\mathrm{H}$ : Cognzyme transport and metabolism

I: Lipid transport and metabolism

$\mathrm{J}$ : Translation, ribosomal structure and biogenesis

K: Transcription

: Replication, recombination and repair

M: Cell wall/membrane/envelope biogenesis

$\mathrm{N}:$ Coll motility

O: Postranslational modification, protein turnover, chaperones

P: Inorganic ion transport and metabolism

Q: Sscondary metabolites biosynthesis, transport and catabolism

R: General function prediction only

S: Function unknown

T: Signal transduction mechanisms

U: Intracellular trafficking, secretion, and vesicular transpor

$\mathrm{v}$ : Dofense mechanisms

$W:$ Extracellular structures

$\gamma:$ Nuclear structure

Z: Cytoskeleton

Fig. 4 KOG function classification of the almond transcriptome. A total of 25 categories were obtained. The $x$-axis indicates the categories, and the $y$-axis indicates the numbers of the unigenes 
were assigned to $129 \mathrm{KEGG}$ pathways that belonged to five categories, namely, metabolic pathways (5648, 59.60\%), genetic information processing $(2636,27.8 \%)$, cellular processes (512, 5.4\%), environmental information processing (389, 4.1\%) and organismal systems (285, 3.0\%) (Additional file 4). The majority of the unigene pathways were associated with ribosomes $(443,7.3 \%)$, carbon metabolism (320, $5.3 \%$, protein processing in the endoplasmic reticulum (290, 4.8\%), biosynthesis of amino acids (277, 4.9\%), spliceosomes (274, 4.5\%), plant hormone signal transduction (249, 4.1\%) and endocytosis (245, 4.0\%).

\section{Development and characterisation of SSR markers}

In this study, the unigene sequences were used to develop new SSR markers with MISA software. A total of 8641 SSRs were identified from 48,012 unigenes. For the 8641 SSRs, di-nucleotide motifs were the most abundant form $(5141,59.5 \%)$, followed by tri-nucleotides (2416, $28.5 \%)$, tetra-nucleotides (606, 7.0\%), hexa-nucleotides (277, 3.2\%) and penta-nucleotides (201, 2.3\%) (Table 2). In addition, the number of repeated units of the dinucleotide motifs ranged from 6 to 15, and the trinucleotide, tetra-nucleotide, penta-nucleotide, and hexanucleotide motifs included 5 to 15,4 to 10,4 to 8 , and 4 to 7 , respectively. SSRs with six tandem repeats were the most frequent $(1795,20.8 \%)$, followed by five tandem repeats $(1543,17.9 \%)$, more than fifteen tandem repeats (1228, 14.2\%), seven tandem repeats $(1095,12.7 \%)$, and others (Table 3). The most frequent motif types of SSRs were AG/CT (49.0\%), followed by AAG/CTT (9.3\%), $\mathrm{AT} / \mathrm{AT}$ (5.9\%), AC/GT (4.5\%), AGG/CCT (3.6\%) and others (Additional file 5).

\section{Cross-species transferability of $A$. communis SSR markers}

One hundred SSR sites were randomly selected to design SSR primers (Additional file 6). Among these 100 primer pairs, 82 could amplify the specific products (these 82 markers are highlighted in red in Additional file 6), while

Table 2 Summary of the EST-SSRr data for almond

\begin{tabular}{ll}
\hline Item & Number \\
\hline Total number of sequences examined & 42,135 \\
Total size of examined sequences (bp) & $41,663,061$ \\
Total number of identified SSRs & 8641 \\
Number of SSR containing sequences & 6780 \\
Number of sequences containing more than 1 SSR & 1433 \\
Number of SSRs present in compound formation & 752 \\
Di-nucleotide & $5141(59.5 \%)$ \\
Tri-nucleotide & $2416(28.5 \%)$ \\
Tetra-nucleotide & $606(7.0 \%)$ \\
Penta-nucleotide & $201(2.3 \%)$ \\
Hexa-nucleotide & $277(3.2 \%)$ \\
\hline
\end{tabular}

the remaining 18 did not generate PCR products. To validate the transferability of $A$. communis SSR markers, five species (A. ledebouriana, A. mongolica, A. pedunculata, A. tangutica, and A. triloba) (Additional file 7) were assessed using the 82 SSR markers selected above. The results indicated that 70 SSR markers were transferable to these five species and that 12 SSR markers did not generate bands. The PCR amplification results of some primers are shown in Fig. 5. The UPMGA cluster analysis indicated that $A$. communis and A. mongolica are more closely related (Fig. 6).

\section{Discussion}

Almonds are one of the most important commercially cultivated crops in subtropical regions, specifically in southwest Asia, the Middle East, and the Mediterranean [8] because almonds have a high nutrient value. Previous studies concerning almonds focused on physicochemical properties, seed nutrients and oil extraction $[2,7,8]$. However, no studies have yet constructed a genetic linkage map, including QTL mapping of important agronomical traits and marker-assisted selection in almond trees. Until now, genome sequencing of some important fruits has been completed using NGS technologies. Transcriptome analysis based on NGS technologies, such as Illumina and 454 sequencing platforms, has provided an efficient tool for obtaining genomic data for some plants without a reference genome, such as wax gourds [21] and pumpkins [22]. Therefore, we sequenced the almond transcriptome to obtain genomic data and then developed many SSR markers. These transcriptome data will provide information for future studies on breeding and molecular biology.

In this study, a large number of transcriptomic unigenes (42135) was obtained using the Illumina HiSeq ${ }^{\mathrm{TM}}$ 2500 platform, and the average unigene length was $988 \mathrm{bp}$. Consistent with recently published plant species, the average length of the unigenes was relatively long (835 bp) compared to black gram (443 bp) [10], caragana (709 bp) [18], wax gourd (709 bp) [21], pumpkin (765 bp) [22], Siberian apricot (652 bp) [23], Chinese jujube (473.4 bp) [24], and safflower (446 bp) [25]. These results indicate a higher quality of almond transcriptome sequencing and de novo assembly. In this study, approximately 27,586 unigenes $(57.7 \%$ of all unigenes) were annotated by BLAST searches of the $\mathrm{Nr}, \mathrm{GO}$, Swiss-Prot, KEGG and KOG databases. Moreover, $12,671(42.5 \%)$ of the unigenes did not annotate to any databases. Technical limitations, such as read length and sequencing depth, may account for these unannotated unigenes. For gene annotation, the sequences of unigenes were blasted against the Nr, Swiss-Prot, KEGG, GO and KOG databases. Approximately 27,586 unigenes (57.7\% of all unigenes) were annotated in four protein 
Table 3 Summary of the different repeat units of identified EST-SSRs

\begin{tabular}{|c|c|c|c|c|c|c|c|}
\hline Number of repeat unit & Di- & Tri- & Tetra- & Penta- & Hexa & Total & Percentage (\%) \\
\hline 4 & 0 & 0 & 435 & 151 & 200 & 786 & 9.1 \\
\hline 5 & 0 & 1321 & 121 & 39 & 62 & 1543 & 17.9 \\
\hline 6 & 1156 & 592 & 31 & 7 & 9 & 1795 & 20.8 \\
\hline 7 & 771 & 302 & 13 & 3 & 6 & 1095 & 12.7 \\
\hline 8 & 596 & 67 & 4 & 1 & 0 & 668 & 7.7 \\
\hline 9 & 566 & 38 & 1 & 0 & 0 & 605 & 7.0 \\
\hline 10 & 439 & 58 & 1 & 0 & 0 & 498 & 5.8 \\
\hline 11 & 237 & 11 & 0 & 0 & 0 & 248 & 2.9 \\
\hline 12 & 38 & 9 & 0 & 0 & 0 & 47 & 0.5 \\
\hline 13 & 5 & 10 & 0 & 0 & 0 & 15 & 0.2 \\
\hline 14 & 109 & 4 & 0 & 0 & 0 & 113 & 1.3 \\
\hline$\geq 15$ & 1224 & 4 & 0 & 0 & 0 & 1228 & 14.2 \\
\hline Total & 5141 & 2416 & 606 & 201 & 277 & 8641 & 100 \\
\hline
\end{tabular}

databases, indicating that the transcriptomic data of almonds may have large transcript diversity. Additionally, approximately 12,671 (42.5\%) unigenes were not annotated to the four databases, suggesting that some unigenes may be unique to almonds.

SSR markers have been important in some research, including the assessment of genetic diversity and genetic relationships, the construction of genetic maps, markerassisted selection of important agronomic traits, and others $[2,26]$. Previous studies have also shown that SSR markers are highly polymorphic, codominant and easily reproducible [9]. Due to the time-consuming and expensive nature of traditional methods for SSR marker development, few SSR markers have been reported for almonds, and no studies have reported the development of SSR markers in A. communis, which has limited the application of SSR markers in almond trees. Transcriptome sequencing is an efficient technology for the development of SSR markers in plants, and the SSR markers for some plants have been reported using transcriptome sequencing [10, 21-25]. Our results produced a large number of transcriptome sequences that could be used to develop SSR markers in almonds. In total, 8641 SSR markers were identified from 48,012 unigenes. In this study, di-nucleotide motifs were the most abundant form, followed by tri-nucleotides, tetra-nucleotides, hexa-nucleotides and penta-nucleotides, which is similar to previous studies [23-25]. In addition, the most abundant di-nucleotides and tri-nucleotides were AG/CT and AAG/CTT, respectively, which was consistent with previous reports [20-24]. To assess the quality of SSR markers, we randomly selected 100 pairs of primers and assessed them in five species. Eighty-two percent showed polymorphisms. This result was similar to results found in other plants. The UPMGA cluster analysis indicated that $A$. communis and A. mongolica were more closely

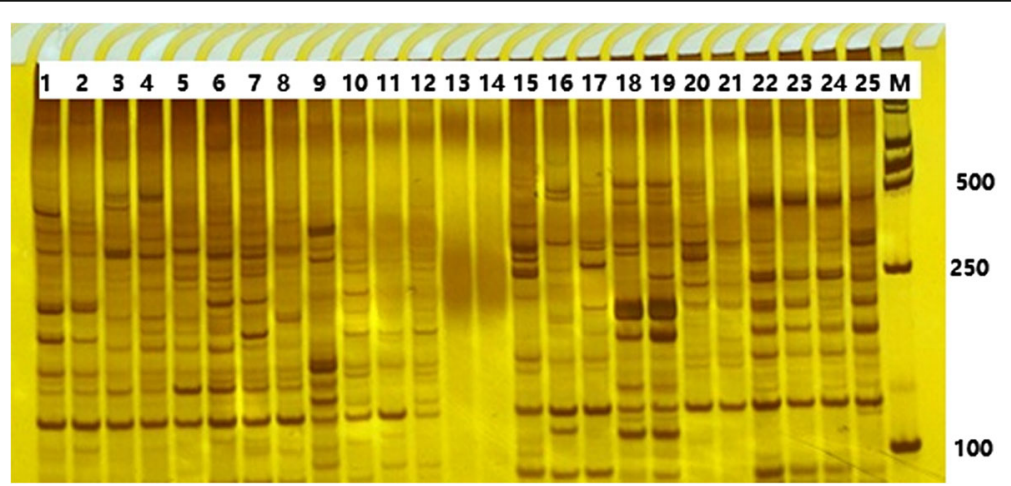

Fig. 5 Examples of polymorphic products amplified by different SSR primer pairs. 1-25 represent different primer pairs. M represents DL2000 DNA markers 


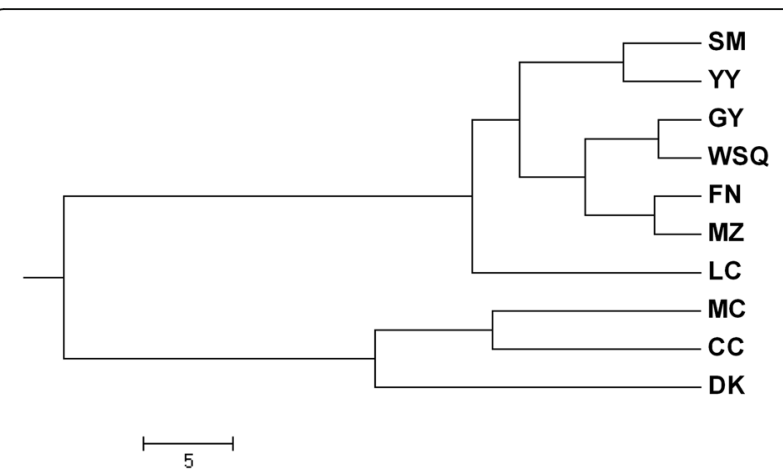

Fig. 6 Dendrogram obtained using UPGMA cluster analysis based on Nei's genetic distances among the ten populations of almonds. The population abbreviations are the same as those in Additional file 7

related, which was consistent with Jing et al., who reported using SRAP markers [2]. We believe that the new SSR markers will be used to study genetic diversity, genetic mapping, and, in particular, marker-assisted breeding for almonds.

\section{Conclusion}

This paper reports on the transcriptome characterizations of almond trees and provides a large number of SSR markers to elucidate the molecular biology of almond trees. To our knowledge, this is the first attempt to develop SSR markers for almonds using a transcriptome sequencing method, and these developed SSR markers will significantly contribute to genetic diversity studies, QTL mapping, and marker-assisted selection breeding for almonds. Notably, due to high transferability, these SSR markers may provide an efficient tool to accelerate molecular breeding in other Amygdalus species.

\section{Methods}

\section{Plant materials and RNA extraction}

Plants of $A$. communis were grown at the experimental farm of Northwest A\&F University, Yangling, China. Tissues from leaves, flowers, stems and fruits were harvested from six individuals. The sampled tissues were immediately frozen in liquid nitrogen and stored at $-80{ }^{\circ} \mathrm{C}$ for later RNA extraction. The RNA samples were isolated using an E.Z.N.A. ${ }^{\bullet}$ Plant RNA Kit (Omega Biotek, Inc.) according to the manufacturer's protocol. The quality and quantity of RNA were assessed using electrophoresis on 1\% agarose gels and a NanoDrop 1000 spectrophotometer (Thermo Scientific, Wilmington, DE, USA), respectively. High-quality RNA was used for further analyses. Equal amounts of RNA from different samples were pooled for further studies.
cDNA library construction and transcriptome sequencing After the RNA was extracted, the cDNA library construction was performed using a TransCript ${ }^{\bullet}$ cDNA sample prep kit (TransGen Biotech, China). The ligation products were size-selected with agarose gel electrophoresis, PCR-amplified, and sequenced using Illumina HiSeqTM 2500 by Gene Denovo Biotechnology Co., Ltd. (Guangzhou, China).

Data filtering, de novo assembly and function annotation Reads obtained from the sequencing machines included raw reads containing adapters or low-quality bases that would affect the following assembly and analysis. Thus, to obtain high-quality clean reads, the clean reads were assembled using the Trinity assembly program [27]. The isoform was obtained using Trinity software. To eliminate redundant information, the longest isoform was taken as the gene to further analyse; this was defined as the unigene. The functional annotation of unigene sequences was performed by BLASTX search of the nonredundant (Nr) (http://www.ncbi.nlm.nih.gov) database, Swiss-Prot protein database, Kyoto Encyclopaedia of Genes and Genomes (KEGG) database (http://www.genome.jp/kegg), Clusters of Orthologous Groups (KOG) database, and Gene Ontology (GO) database with an Evalue $<10-5$. We used the Blast2GO program to analyse the GO annotations of the unigenes [28]. The functional classifications were determined with WEGO software [29]. Pathway information of unigenes was collected from KEGG databases [30].

\section{SSR detection and primer design}

MISA software (http://pgrc.ipk-gatersleben.de/misa/misa.html) was used to identify microsatellites in the whole transcriptome. The parameters were as follows: definition (unit_size, min_repeats): 2-6 3-5 4-4 5-4 6-4; interruptions (max_difference_between_2_SSRs): 100. If the distance between two SSRs was shorter than $100 \mathrm{bp}$, they were considered to be one SSR. Based on the MISA results, primer pairs of each SSR loci were designed using Primer premier 3.0 (PREMIER Biosoft International, Palo Alto, CA) in the flanking regions of SSRs.

\section{Validation of SSR markers}

To validate the SSR markers, a total of 100 primer pairs were randomly selected and synthesized. Wild almond germplasm from six species $(A$. communis, $A$. ledebouriana, A. mongolica, A. pedunculata, A. tangutica, and $A$. triloba) were used to validate the SSR markers. The total genomic DNA was extracted from fresh leaves using a plant DNA extraction kit (TIANGEN', China). The DNA quality and concentration were tested with a NanoDrop ND 1000 spectrophotometer (Thermo Scientific, USA). PCR amplification reactions 
were performed in a $25 \mu \mathrm{L}$ volume, containing $40 \mathrm{ng}$ of DNA, $0.2 \mathrm{mM}$ dNTPs, $1.5 \mathrm{pM}$ aliquots of forward and reverse primers, $2.5 \mathrm{mM} \mathrm{Mg}^{2+}, 1 \mathrm{U}$ Taq DNA polymerase (TaKaRa Biotechnology Dalian Co., Ltd., China), and $1 \times$ Taq Buffer (10 mM Tris- $\mathrm{HCl}, \mathrm{pH} 8.3,50 \mathrm{mM}$ $\mathrm{KCl})$. PCR amplification was performed with the following conditions: initial denaturation at $94{ }^{\circ} \mathrm{C}$ for $5 \mathrm{~min} ; 30$ cycles at $94{ }^{\circ} \mathrm{C}$ for $30 \mathrm{~s}$, a primer-specific annealing temperature for $60 \mathrm{~s}$, and $72{ }^{\circ} \mathrm{C}$ for $90 \mathrm{~s}$; and $72{ }^{\circ} \mathrm{C}$ for $7 \mathrm{~min}$. PCR products were separated by electrophoresis on denaturing 6\% polyacrylamide gels and visualized using silver staining. The molecular size of the amplified fragments was estimated using a 10-bp DNA ladder (TransGen Biotech, China).

\section{Additional files}

Additional file 1: The coverage percentage of reads blasted unigenes. (PNG $25 \mathrm{~kb}$ )

Additional file 2: Frequency distribution of SSRs from different motif types. (XLSX $11 \mathrm{~kb}$ )

Additional file 3: The gene ontology classification of assembled unigenes. (XLSX $10 \mathrm{~kb})$

Additional file 4: The details of KOG classifications. (XLSX $14 \mathrm{~kb}$ )

Additional file 5: The $129 \mathrm{KEGG}$ pathway annotations. (PNG $11 \mathrm{~kb}$ )

Additional file 6: SSR sites developed in this study. (XLSX $1402 \mathrm{~kb}$ )

Additional file 7: List of species in this study. (DOCX $16 \mathrm{~kb}$ )

\section{Abbreviations}

GO: Gene Ontology; KEGG: Kyoto Encyclopaedia of Genes and Genomes; KOG: Clusters of Orthologous Groups; Nr: non-redundant; SSR: simple sequence repeats marker

\section{Acknowledgements}

We would like to thank AJE (https://www.aje.com/) for their expert language editing of this manuscript.

\section{Funding}

This work was supported by the earmarked fund for China Agriculture Research System (CARS-28), China Postdoctoral Science Foundation (2015 M582712), the Postdoctoral Science Foundation of Shaanxi Province (2016BSHYDZZ07), and the Science and Technology Research and Development Program of Shaanxi Province (2015KTZDNY02-03-01, 2015KJZDNY02-03-02, 2016KJXX-58).

\section{Availability of data and materials}

The data are available at https://www.ncbi.nlm.nih.gov/.

\section{Authors' contributions}

$\mathrm{L}-\mathrm{SZ}$ and Z-BJ conceived the project and designed the experiments. X-NY, X$\mathrm{NQ}$, and C-HG performed the experiment. L-SZ, and Z-BJ wrote and revised the paper. All authors discussed the results and commented on the manuscript. All authors read and approved the final manuscript.

\section{Ethics approval and consent to participate}

Not applicable.

\section{Consent for publication}

Not applicable.

\section{Competing interests}

The authors declare that they have no competing interests.

\section{Publisher's Note}

Springer Nature remains neutral with regard to jurisdictional claims in published maps and institutional affiliations.

Received: 5 December 2016 Accepted: 9 October 2017

Published online: 19 October 2017

\section{References}

1. Sorkheh K, Dehkordi MK, Ercisli S, Hegedus A, Julia H. Comparison of traditional and new generation DNA markers delares high genetic diversity and differentiated population structure of wild almond species. Sci Rep. 2017;7:5966

2. Jing ZB, Cheng JM, Guo CH, Wang XP. Seed traits, nutrient elements and assessment of genetic diversity for almond (Amygdalus spp.) endangered to China as revealed using SRAP markers. Bioche Syst Ecol. 2013:49:51-7.

3. Arman MO, Mohammad RG. Assessment of genetic diversity in late flowering almond varieties using ISSR molecular markers aimed to select genotypes tolerant to early spring frost in Yazd province. Curr Bot. 2011;2(1):01-4.

4. Gradziel T, Martinez-Gomez P, Dicenta F, Kester D. The utilization of related Prunus species for almond variety improvement. J Am Pomo Soc. 2001;55:100-8.

5. Li N, Xia X, Chen C, Blumberg J, Song Y, Zhang W, Zhang X, et al. Almond consumption reduces oxidative DNA damage and lipid peroxidation in male smokers. J Nutr. 2007;137:2717-22.

6. Omirshat T, Geng YP, Zeng LY, Dong SS, Chen F, Chen J, Song ZP, et al. Assessment of genetic diversity and population structure of Chinese wild almond, Amygdalus nana, using EST and genomic SSRs. Biochem Sys Ecol. 2009;37:46-153.

7. Balvardi M, Mendiola J, Castro-Gomez P, Fontecha J, Rezaei K, Ibanez E. Development of pressurized extraction processes for oil recovery from wild almond (Amygdalus scoparia). J Am Oil Chem Soc. 2015;92:1503-11.

8. Rezaei A, Nasirpour A, Tavanai H. Fractionation and some physicochemical properties of almond gum (Amygdalus communis L.) exudates. Food Hydrocoll. 2016;60:461-9.

9. Yang $Y$, Jing ZB, Ruan $X$, Cheng JM. Development of simple sequence repeat markers in persimmon (Diospyros L.) and their potential use in related species. Genet Mol Res. 2015;14(1):609-18.

10. Souframanien J, Reddy KS. De novo assembly, characterization of immature seed Transcriptome and development of Genic-SSR markers in black gram (Vigna Mungo L.) Hepper. PLoS One. 2015;10(6):e0128748.

11. Forcada CF, Marti AF, Company RS. Mapping quantitative trait loci for kernel composition in almond. BMC Genet. 2012;13:47.

12. Tavassolian I, Rabiei G, Gregory D, Mnejja M, Wirthensohn MG, Hunt PW, Gibson JP, Ford CM, Sedgley M, Wu SB. Construction of an almond linkage map in an Australian population nonpareilXLauranne. BMC Genet. 2010;11:551

13. Forcada CFI, Oraguzie N, Reyes-Chin-Wo S, Espiau MT, Company RSI, Marti AFI. Identification of genetic loci associated with quality traits in almond via association mapping. PLoS One. 2015;10(6):e0127656.

14. Hassouna G, Elhem K, Tatiana BZ, Ahmed M, Yolanda G. Development of an SRR-based identification key for Tunisian local almonds. Sci Agric. 2012:69(2):108-13.

15. Mnejja M, Garcia-Mas J, Howad W, Arus P. Development and transportability across Prunus species of 42 polymorphic almond microsatellites. Mol Ecol Notes. 2005:5(3):531-5

16. Xie H, Sui $Y$, Chang FQ, Xu Y, Ma RC. SSR allelic variation in almond (Prunus Dulcis mill.). Theor Appl Genet. 2006;112(2):366-72.

17. An M, Deng M, Zheng SS, Song YG. De novo transcriptome assembly and development of SSR markers of oaks Quercus austrocochinchinensis and Q. Kerrii (Fagaceae). Tree Genet Geno. 2016;12(103):1-9.

18. Long $Y$, Wang $Y$, Wu S, Wang J, Tian $X$, Pei $X$. De novo assembly of Transcriptome sequencing in Caragana Korshinskii Kom. And characterization of EST-SSR markers. PLoS One. 2015;10(1):e0115805.

19. Chi W, Ma XF, Niu JG, Zou M. Characterizing the transcriptome and molecular markers information for roach. J Genet. 2016;95:45-51.

20. Zhou XJ, Wang YY, Xu YN, Yan RS, Zhao P, Liu W. De novo characterization of flower bud Transcriptomes and the development of EST-SSR markers for the endangered tree Tapiscia Sinensis. Int J Mol Sci. 2015;16:12855-70.

21. Jiang B, Xie D, Liu W, Peng Q, He X. De novo assembly and characterization of the Transcriptome and development of SSR markers in wax gourd (Benicasa hispida). PLoS One. 2013;8(8):e71054. 
22. Wu TQ, Luo SB, Wang R, Zhong YJ, Xu XM, Lin TE, He XM, et al. The first Illumina-based de novo transcriptome sequencing and analysis of pumpkin (Cucurbita Moschata Duch.) and SSR marker development. Mol Breeding. 2014:34:1437-47.

23. Dong S, Liu Y, Niu J, Ning Y, Lin S, Zhang Z. De novo transcriptome analysis of the Siberian apricot (Prunus Sibirica L.) and search for potential SSR markers by 454 pyrosequencing. Gene. 2014;544:220-7.

24. Li Y, Xu C, Lin X, Cui B, Wu R, Pang X. De novo assembly and characterization of the fruit transcriptome of Chinese jujube (Ziziphus Jujuba mill.) using 454 pyrosequencing and the development of novel tri-nucleotide SSR markers. PLoS One. 2014;9:e106438.

25. Huang LL, Yang X, Sun P, Tong W, Hu SQ. The first Illumian based de novo transcriptome sequencing and analysis of safflower flowers. PLoS One. 2012;7:e38653.

26. Ahmad Z, Mumtaz AS, Ghafoor A, Ali A, Nisar M. Marker assisted selection (MAS) for chickpea Fusarium oxysporum wilt resistant genotypes using PCR based molecular markers. Mol Biol Rep. 2014:41:6755-62.

27. Grabherr MG, Haas BJ, Yassour M, Levin JZ, Thompson DA, Amit I, Adiconis $Z$, et al. Full-length transcriptome assembly from RNA-Seq data without a reference genome. Nat Biotechnol. 2011;29:644-52.

28. Conesa A, Gotz S, Garcia-Gomez JM, Terol J, Talon M, Robles M. Blast2GO, a universal tool for annotation, visualization and analysis in functional genomics research. Bioinformatics. 2005;21(18):3674-6.

29. Ye J, Fang L, Zheng H, Zhang Y, Chen J, Zhang Z, Wang J, Li S, et al. WEGO, a web tool for plotting GO annotations. Nucleic Acids Res. 2006;34:W293-7.

30. Kanehisa M, Araki M, Goto S, Hattori M, Hirakawa M, Itoh M, Katayama T, et al. KEGG for linking genomes to life and the environment. Nucleic Acids Res. 2008;36:D480-4.

\section{Submit your next manuscript to BioMed Central and we will help you at every step:}

- We accept pre-submission inquiries

- Our selector tool helps you to find the most relevant journal

- We provide round the clock customer support

- Convenient online submission

- Thorough peer review

- Inclusion in PubMed and all major indexing services

- Maximum visibility for your research

Submit your manuscript at www.biomedcentral.com/submit 